\title{
Bjomédica
}

Articulo Original

\section{Características sociodemográficas y perfil de consumo de tabaco y drogas en estudiantes de dos universidades de México}

Sánchez-Hoil Alejandro (1), Andueza-Pech María Guadalupe (1) Santana-Carvajal Andrés Martín (1), Hoil-Santos Jolly Josefina (1), CuFarfán-López Julio (2)

(1) Centro de Investigaciones Regionales “Dr. Hideyo Noguchi”. Universidad Autónoma de Yucatán

(2) Facultad de Ciencia, Educación y Humanidades. Universidad Autónoma de Coahuila

\section{Autor para correspondencia}

Nombre: Alejandro Sánchez Hoil

Institución: Centro de Investigaciones Regionales "Dr. Hideyo Noguchi”"

E-mail: sanchezhalex@gmail.com

Recibido: el 28 de abril de 2016 Aceptado para publicación: el 29 de septiembre de 2016

Copyright @ 2016 por autores (s) y Revista Biomédica.

Este trabajo esta licenciado bajo las atribuciones de la Creative Commons (CC BY).

http://creativecommons.org/licenses/by/4.0/

(c) (i) Open Access 
Sánchez-Hoil et al.

Consumo de tabaco y drogas en estudiantes de dos universidades de México

\section{Resumen}

Introducción. El consumo de tabaco es la primera causa prevenible de muerte prematura y enfermedad en todo el mundo; asimismo el uso y abuso de drogas constituye un grave problema de salud pública y de altos costos de atención a la salud. Se aborda el consumo de tabaco y drogas, desde el punto de vista epidemiológico y en materia de salud pública, así como los factores sociodemográficos que influyen en la adicción a ellos.

Objetivo. Describir las características socio-demográficas y de consumo de tabaco y droga en estudiantes universitarios de Yucatán y Coahuila.

Material y métodos. Encuesta transversal que incluyó 412 universitarios de Yucatán y 344 de Coahuila. Se utilizó estadística descriptiva para las variables numéricas y frecuencias para las cualitativas, estadística inferencial para las coincidencias y diferencias.

Resultados. La media de edad fue mayor para los estudiantes de Yucatán así como predominio de los hombres, la media de consumidores de tabaco dentro de las personas de su red fue mayor para Coahuila siendo las diferencias estadísticamente significativas $\mathrm{p}<0.05$, no así para las personas de la red respecto al consumo de drogas.

Conclusiones. El consumo de tabaco en ambos estados fue mayor al esperado para su región, las fumadoras activas estuvieron por encima del promedio nacional, los dos estados se ubicaron arriba del promedio nacional respecto a consumo de drogas y Coahuila por encima del promedio mundial. Se propone un trabajo conjunto para evaluar los spots publicitarios transmitidos en los medios de comunicación y evitar su incorporación al mercado como bienes de consumo.

Palabras Clave: Características sociodemográficas, tabaco, drogas, perfil de consumo, universitarios.

\section{Abstract}

\section{Tobacco and drugs consumption in students from two universities in Mexico.}

Introduction: Tobacco consumption is the leading cause of premature preventable death and disease worldwide, as well the drug use and abuse is a serious public health problem and health care high costs. The tobacco and drugs consumption is addressed from the 
epidemiologic and public health point of view, also the socio-demographic factors influencing addiction to them

Objetive: Describe the sociodemographic and tobacco and drugs consumption characteristics among college students from Yucatan and Coahuila

Materials and methods: Cross sectional survey that included 412 college students from Yucatan and 344 from Coahuila. Descriptive statistics were used for the numeric variables, frequencies for the qualitative, inferential statistics for the similarities and differences.

Results: The mean of the age was higher for Yucatan students and men predominated, the mean of the tobacco consumers within people from their social network was higher for Coahuila being the differences statistically significant $\mathrm{p}<0.05$, not so for the people from their network regarding to drugs consumption

Conclusions: The tobacco consumption in both states was higher than expected for the region, the active female smokers were above the national mean, the two states were above the national mean regarding to drugs use and Coahuila above the world mean. Joint work is proposed to evaluate the advertising spots broadcast in the media and avoid its incorporation to the market as consumer goods.

Keywords: Sociodemographic characteristics, tobacco, drugs, consumption profiles, college students 
Sánchez-Hoil et al.

Introducción

La adicción a drogas licitas e ilícitas constituye un problema de salud pública en la actualidad que se presenta, tanto a nivel nacional como internacional. Este fenómeno de salud afecta sin distinción de género e incide principalmente en niños y adolescentes, de cualquier estrato social y de todas las regiones de nuestro país. Los estudios epidemiológicos, tanto mundiales como nacionales demuestran que este suceso es cambiante y actualmente presenta un incremento de la incidencia en jóvenes, además de un aumento en el uso de drogas en la mujer, tanto legales como ilegales $(1,2)$.

En el contexto internacional, México es uno de los países con bajo nivel de consumo de drogas, pero a su vez, reporta un incremento si se consideran las tendencias epidemiológicas más recientes. Este problema afecta de manera específica a la población de niños, adolescentes y jóvenes, quienes son particularmente vulnerables debido a que, al encontrarse en pleno desarrollo, cualquier exposición ante el consumo de tabaco, alcohol y otras drogas, incrementa significativamente la posibilidad de que se inicien en las adicciones y presenten problemas que afecten su salud, su desempeño escolar y su proyecto de vida en general (3).

El Sistema de Vigilancia Epidemiológica de las Adicciones (4) reporta, que el consumo de alcohol y tabaco son las puertas de entrada para el consumo de drogas ilícitas. La OMS afirma que la epidemia del tabaquismo ha sido catalogada como un problema de salud pública de importancia internacional cuyo crecimiento se ha transformado en un reto para los sistemas nacionales de salud ya que impacta negativamente en la economía nacional, en la productividad laboral por mortalidad prematura y eleva los costos en la oferta de servicios médicos (5). El consumo de tabaco es la primera causa prevenible de muerte prematura y morbilidad en todo el mundo. A nivel mundial se producen aproximadamente 6 millones de muertes por año y las tendencias indican que causarán más de 8 millones de muertes de manera anual para 2030 (5). Fumar tabaco es la forma de administración de la nicotina más extendida por todo el mundo. Los métodos más habituales de fumar tabaco son: el cigarrillo, el cigarro puro y la pipa. Se absorbe rápidamente por la mucosa nasal, oral y respiratoria y llega al cerebro donde están los receptores al cabo de unos 7 segundos. Esta relación casi inmediata entre la inhalación del humo y su efecto a nivel cerebral es uno de los factores que contribuye al alto poder adictivo de la nicotina (6).

Respecto a drogas ilícitas, la Encuesta Nacional de Adicciones 2011 (1) reportó para México, una prevalencia del consumo de drogas ilegales en la población de 12 a 65 años de 1.8\%; la dependencia a las drogas afecta más a hombres $(1.3 \%)$ en comparación con las mujeres $(0.2 \%)$. El cannabis es la sustancia ilícita más consumida en México y en el mundo. La prevalencia de consumidores de 
cannabis es de 180,6 millones, es decir, el 3,9\% de la población de 15 a 64 años de edad a nivel mundial. En México su prevalencia es del 1.2\%. La cocaína es la segunda droga de mayor prevalencia en el país con $0.5 \%$. La población más afectada es la de los jóvenes, la edad promedio del inicio del consumo de drogas es de 18.8 años, los hombres inician su consumo 2 años antes que las mujeres. Se ha visto que cada vez inician su consumo a edades más tempranas, ya que la edad promedio de inicio disminuyó entre 2002 y 2008; en las mujeres paso de 23.6 años a 20.1 años, en tanto que en los hombres de 19.8 años a 18.3 años. Además de que el porcentaje de consumo de mujeres ha ido en aumento, repercutiendo ello en los índices de morbilidad y mortalidad con un alto costo social, laboral y familiar (7- 10).

En el campo de la salud, la juventud guarda un lugar relevante en la generación de conocimiento y en el destino de recursos de política pública. Principalmente porque se mira a la juventud como factor de riesgo, con patologías diversas y pasivo ante las necesidades de cuidado para generar procesos de salud responsable. Fundamentalmente en el terreno de la salud pública por muchos años la juventud ha sido estudiada en el ámbito de la salud reproductiva, en búsqueda de controlar la reproducción y las prácticas de riesgo de infecciones de transmisión sexual, con preponderancia en el contagio del VIH-SIDA en los últimos tiempos. Al paso del tiempo, aunadas a estas preocupaciones se suma el tema de la violencia en el noviazgo, la violencia de género así como las adicciones (11).

El estudio del análisis de las adicciones desde el ámbito universitario constituye una oportunidad para desarrollar estrategias de prevención acorde a las características de los estudiantes que la conforman, a su filosofía, al ámbito social, político y económico que se cuenta. Con la cual se busca brindar una oferta educativa que incluya la creación de políticas públicas para la promoción de entornos saludables y libres del consumo de sustancias adictivas para el beneficio de los jóvenes de México.

En el análisis de los comportamientos hacía las conductas adictivas (drogas lícitas e ilícitas) un elemento a considerar son los contextos particulares que permean las respuestas a campañas elaboradas a nivel poblacional sobre todo cuando se trata de personas que están en formación de criterios y conductas de vida como son los adolescentes y jóvenes universitarios. Para ello, resulta de gran importancia conocer las tendencias actuales de este grupo poblacional en dos regiones de la república mexicana que den cuenta de la problemática de las adicciones: Yucatán y Coahuila.

En su informe 2011, la ENA consideró 7 regiones y el Distrito Federal. Coahuila se ubica en la región norte centro y Yucatán en la región sur. En el caso específico de Coahuila se estimó que 41.3\% de la población de 20 años de edad y más, ha fumado más de 100 cigarrillos en toda su vida, con un porcentaje mayor en hombres $(62.6 \%)$ que en mujeres (21.0\%), y una razón hombre:mujer de 3. En 
Sánchez-Hoil et al.

cuanto a Yucatán se refiere, se estimó que 26.7\% de la población de 20 años de edad o más, ha fumado más de 100 cigarrillos en toda su vida, con un porcentaje mayor en hombres (46.3\%) que en mujeres (8.5\%), y una razón hombre:mujer de 5.4 (12).

De acuerdo al informe del Sistema de Vigilancia Epidemiológica de las Adicciones (SISVEA) 2011 (4), en Coahuila se observó que el tabaco fue la principal droga de inicio con el 35.1\%, seguida por el alcohol y la marihuana con $29.4 \%$ y $20.5 \%$ respectivamente. En esta zona, $31.2 \%$ de las personas que acudieron a centros de tratamiento, refirieron como droga de impacto el cristal, seguido del alcohol (22.5\%) y la heroína (15.6\%) (13).

En tanto que en Yucatán se reportó que la marihuana tiene el primer lugar con una prevalencia de $10.85 \%$ como droga de inicio y $15.4 \%$ como droga de impacto, representando el $80 \%$ del consumo total de las drogas ilegales; en segundo lugar está la cocaína con un usuario de cocaína por cada 2.4 de marihuana (13).

Si retomamos lo dicho en párrafos anteriores acerca de la importancia de considerar los contextos particulares para analizar el comportamiento de las conductas adictivas en los jóvenes y después de mirar las tendencias de estás dos problemáticas en estos dos estados, entonces debemos aceptar que se requiere de una mirada múltiple para comprender el fenómeno de las adicciones, sobre todo en la población joven. Como plantea Rodríguez (14) es necesario tomar en cuenta las redes sociales así como los actores sociales que están involucrados en este proceso; no menos importante el ambiente social en el que está inmersa la población juvenil y mucho más los universitarios, ello, además de su perfil sociodemográfico.

Existen pocos estudios respecto de los factores sociales y demográficos que influyen en el consumo de tabaco en la población joven, sin embargo uno realizado en Barcelona en estudiantes de nivel secundaria en el 2001 reveló que en los chicos, los determinantes del consumo son la norma social (odds ratio $[\mathrm{OR}]=2,5$; intervalo de confianza [IC] del 95\%, 1,2-5,2), el consumo de tabaco por parte de los hermanos (OR = 2,4; IC del 95\%, 1,1-5,2), las actitudes contrarias al consumo (OR = 0,3; IC del 95\%, 0,1-0,6), la práctica de deporte en el tiempo libre (OR =0,3; IC del 95\%, 0,1-0,7) y el dinero semanal disponible (OR = 3,2; IC del 95\%, 1,5-6,8), mientras que la intención únicamente se asoció de forma significativa a las actitudes contrarias (OR =0,3; IC del 95\%, 0,2-0,5). Para las chicas, el consumo se asoció a la presión (OR = 2,5; IC del 95\%, 1,2-5,0) el consumo por parte de los amigos $(\mathrm{OR}=6,0$; IC del 95\%, 2,4-15,4) y las actitudes contrarias (OR = 0,2; IC del 95\%, 0,1- 0,4), mientras que la intención se halla asociada a las actitudes contrarias (OR = 0,4; IC del 95\%, 0,2-0,6) y pasar tiempo libre en la calle (OR = 2,1; IC del 95\%, 1,3-3,5) (15). En México se realizó un estudio para 
conocer los determinantes de consumo de alcohol y tabaco en el 2002 que reveló lo siguiente: la estimación conjunta de las dos propensiones de consumo confirmó que ambas decisiones se relacionan en forma estrecha. La existencia de otros jóvenes mayores que fuman o que ingieren alcohol se asoció positiva y significativamente con la probabilidad de que los adolescentes de 12 a 15 años consuman ambas sustancias. A mayor edad, la probabilidad de consumir ambas sustancias adictivas es mayor, sobre todo en los hombres. Por otro lado, los jóvenes que manifestaron vivir con ambos padres tuvieron menor probabilidad de fumar o beber alcohol que aquellos que no vivían con ninguno (16).

Los cambios sociales ocurridos en las últimas décadas, derivados de variaciones en la dinámica y composición de la estructura demográfica de la población, las recurrentes crisis económicas y una acelerada apertura social y cultural frente al proceso de modernización y globalización, han afectado las vidas de las personas en los ámbitos individual, familiar y social en que interviene el abuso de sustancias (17).

Esta acelerada apertura social y cultural acarrea inestabilidad y nuevos valores orientados al consumo y al debilitamiento de los controles sociales tradicionales. En este contexto, el alcohol y las drogas se convierten en bienes de consumo y se incorporan al mercado. Y si bien los países ricos tienen tasas de consumo más altas y, dentro de éstos, los grupos de población con más recursos, el impacto entre los pobres también es mayor, ya que a los problemas inherentes a la pobreza se suman los derivados del abuso de sustancias cuando se dispone de menos recursos para atenderlos. De acuerdo con la Organización Mundial de la Salud y el Banco Mundial, en los países de economía de mercado el abuso de tabaco, alcohol y drogas dan cuenta, respectivamente, del 11.7\%, $10.3 \%$ y $2.3 \%$, de los días de vida sana perdidos por muerte prematura o por discapacidad. A su vez, en los países en vías de desarrollo, la proporción alcanzada es considerablemente menor, $1.4 \%, 2.7 \%$ y $0.4 \%$, respectivamente; Latinoamérica comparte problemas inherentes al subdesarrollo, así como altas tasas de problemas derivados del abuso del alcohol y las drogas, con 9.7\% y $1.6 \%$ días de vida sanos perdidos (18).

Tanto el consumo de tabaco como el consumo de drogas son problemas de salud pública de gran importancia dada la cantidad de muertes atribuibles anuales alrededor del mundo (6millones para el tabaco y 247 mil para las drogas) y el hecho de que estas muertes son en su gran mayoría prevenibles, si se identifica de manera oportuna a los grupos de riesgo así como a los factores que influyen en el consumo y se realizan de modo adecuado las medidas preventivas. Entre los factores que se encuentran involucrados en el inicio de consumo, abuso y adicción a sustancias como el tabaco y las drogas se encuentran factores sociodemográficos entre los cuales se incluyen entre otros: edad, sexo, 
Sánchez-Hoil et al.

escolaridad, estado civil, lugar de residencia y relaciones interpersonales de impacto como son familia y amigos. Por ello, el presente trabajo tuvo como objetivo describir el perfil sociodemográfico de estudiantes universitarios de dos estados de México según su estatus de consumo de tabaco y/o droga.

Material y métodos.

Se realizó un estudio transversal comparativo. La población incluyó a estudiantes universitarios de dos estados de la república mexicana; mediante un muestreo por conveniencia y manteniendo la proporción promedio de las licenciaturas del ciclo escolar 2013-2014 con un intervalo de confianza del $95 \%$ y error del 5\%, se conformó la muestra con 412 universitarios para Yucatán y 344 para Coahuila. Para la recolección de datos se utilizó un cuestionario previamente validado, utilizado en el proyecto Educación y Desarrollo Humano a partir de la Calidad de Vida y la Salud. La información que se presenta corresponden al apartado de datos generales: características socio-demográficas y de consumo de tabaco y droga. El análisis estadístico incluyó estadística descriptica con el cálculo de medias y desviaciones estándar, así como frecuencias. Según el tipo de variable se utilizaron las siguientes pruebas: $t$ de student para las variables cuantitativas y $x^{2}$ para las cualitativas. Los programas estadísticos aplicados fueron SPSS versión 17 y EPI INFO versión 7.

Resultados. Se incluyeron a 467 universitarios yucatecos (YUC) y 344 coahuilenses (COAH) con edades promedio de 20.64 (DS 2.11) y 19.55 (DS 3.87) respectivamente, diferencia que resultó significativa $(\mathrm{p}<0.01)$. En la distribución por sexo hubo predominio de hombres para Yucatán y de mujeres para Coahuila ( $\mathrm{p}<0.01$ ). Acerca del estado civil, más del $90 \%$ de alumnos en los dos estados reportaron ser solteros, los porcentajes diferentes fueron para los que señalaron ser casados o en unión libre (YUC 2.1\%, COAH 6.8\% . p<0.01). El promedio de personas de la red de los jóvenes que fue estadísticamente diferente es para los consumidores de tabaco ( $\mathrm{p}<0.01)$ (cuadro 1).

De acuerdo al estatus de consumidor de tabaco los porcentajes de fumador activo (YUC 19.5\%, COAH 28.2\%) y exfumador (YUC 10.9\%, COAH 15.6\%) fueron mayores para los de Coahuila, en tanto que, de los que nunca han fumado fue mayor para los yucatecos ((YUC 69.6\%, COAH 56.2\%, $\mathrm{p}<0.01)$; por sexo, la distribución del estatus en general se mantiene en los hombres $(\mathrm{p}<0.01)$, mientras que en las mujeres, las exfumadoras $(22 \%)$ y las que nunca han fumado (70\%) los mayores porcentajes fueron para las yucatecas $(\mathrm{p}>0.05)$. El promedio de cigarrillos por día, tanto en fumadores activos como exfumadores fue mayor en hombres y mujeres así como de manera global en universitarios de Coahuila, con diferencias significativas $(\mathrm{p}<0.01)$, a excepción de las mujeres fumadoras activas y exfumadoras ( $>0.05$ ). La salud destacó como la razón principal por la que dejaron de consumir cigarrillos (YUC 66.6\%, COAH 71.6\%), y fue coincidente para los alumnos de 
Yucatán y Coahuila ( $\mathrm{p}>0.05)$. La edad de inicio del consumo de cigarrillos para fumadores activos y exfumadores fue mayor para los alumnos de yucatecos (media YUC 17.18, media COAH 16.15, $\mathrm{p}<0.01$ ), este comportamiento se mantuvo tanto en hombres como en las mujeres $(\mathrm{p}<0.01)$ (Cuadro 2).

De las características sociodemográficas sobresale que la media de edad de los fumadores activos y de los que nunca han fumado es menor para los estudiantes de Coahuila (media 19.62 y $19.1 ; \mathrm{p}<.0 .01$ ). Por sexo se observa que tanto en los fumadores activos como en los exfumadores y los que nunca han fumado, los mayores porcentajes son para los hombres yucatecos y las mujeres de Coahuila, con diferencia estadística solo en el grupo de los que nunca han fumado ( $\mathrm{p}<.0 .01)$. Respecto al estado civil, predominaron los solteros en los diferentes estatus de consumidores. El número de personas de su red que consume tabaco es mayor para los de Coahuila en los tres estatus ( $p<0.01$ ). Cabe señalar que de la red de jóvenes, las personas que consumen tabaco aparecen con mayor frecuencia los amigos (YUC $82.4 \%$ y COAH 76\%), los primos (YUC 40.7\% y COAH 60.4\%) y en tercer lugar el padre (YUC $47.3 \%$ у COAH $44.8 \%$ ) (Cuadro 3).

Con relación al estatus de consumidor de droga los porcentajes de consumidor activo (YUC 5.8\%, COAH 9.7\%) y exconsumidor (YUC 6.6\%, COAH 8.2\%) también fueron mayores para los de Coahuila, no así para los que nunca han consumido drogas que fue mayor para los yucatecos (YUC $87.6 \%$, $\mathrm{COAH} 81.5 \%$ ), comportamiento que no resultó significativo. En la lectura de los datos por sexo esta tendencia se mantiene similar para ambos estados en los diferentes estatus. Respecto al tipo de droga el $100 \%$ de los consumidores activos y más del $90 \%$ de los exconsumidores de ambos estados reportó el consumo de marihuana. Nuevamente la salud aparece como el principal motivo para dejar de consumir drogas al ser referido por el $83.8 \%$ de los estudiantes yucatecos y el $46.4 \%$ de los coahuilenses $(\mathrm{p}<0.01)$. En cuanto a la edad de inicio de consumidores activos y exconsumidores se observa que los de Coahuila inician a menor edad, tanto de manera general como por sexo; con diferencia estadísticamente significativa (cuadro 4).

En la descripción de las características sociodemográficas del grupo de estudiantes consumidores de droga tanto activos como exconsumidores destaca que la edad promedio es menor para los de Coahuila (YUC 20.22, COAH 19.51, p<0.01 y YUC 20.61, COAH 19.5 p >0.05) respectivamente; Por sexo sobresalen las mujeres coahuilenses en el grupo de consumidores activos con el $51.5 \%$ y los hombres yucatecos con el 66.7\%; diferencia que no fue significativa ( $p>0.05)$; este comportamiento fue similar en el grupo de exconsumidores y también entre los que señalaron que nunca han consumido drogas, solo que en éste último grupo la diferencia fue significativa $(\mathrm{p}<0.01)$. Del estado civil predominó el de soltero en todos los estatus para ambos estados. Con respecto al número de 
Sánchez-Hoil et al.

personas de su red que consumen drogas las medias fueron similares $(\mathrm{p}>0.05)$. De nuevo entre las personas que aparecen como consumidores de droga en la red de los estudiantes resaltan los amigos en un 77.8 y $69.7 \%$ y los primos $40.7 \%$ y $45.5 \%$ para los estudiantes yucatecos y los coahuilenses respectivamente (cuadro 5).

\section{Discusión}

Es indudable que en el comportamiento de jóvenes estudiantes acerca del consumo de substancias tanto lícitas como ilícitas, intervienen diversos elementos que tienen que ver con toda una cuestión cultural, social económica y sobre todo de identidad; el papel que juega el contexto y los actores sociales en la vida de los jóvenes puede ser determinante para que desarrollen ciertas prácticas de riesgo que finalmente afectarán su salud, su desempeño escolar y su proyecto de vida en general como señala Villatoro (3).

Hemos visto a lo largo de la descripción de los resultados como la presencia de estas adicciones puede ser una respuesta al inicio de rutinas y exigencias académicas que coinciden con otras exigencias personales y familiares en esta etapa de la vida al confirmar la presencia de las adicciones a edades cada vez más tempranas, es decir, al comienzo de la vida universitaria o incluso previamente. Así lo muestran nuestros resultados al compararlos con los reportes a nivel nacional lo que indica que los porcentajes en el consumo de tabaco se han incrementado, sobre todo para los estudiantes de Coahuila (7). Otro aspecto evidente es el hecho de que el porcentaje de mujeres fumadoras activas también es superior a los datos nacionales independiente de la zona geográfica, esto es, tanto para Yucatán como para Coahuila (7); sin embargo, en cuanto a la población exfumadora el porcentaje es menor para ambos estados respecto de los datos nacionales (19). No menos importante fue notar también que la edad de inicio del consumo de tabaco es cada vez menor $(7,20)$. El hecho de que la población de exfumadores sea menor es algo esperado ya que el abandono del hábito se correlaciona a mayor edad y la población del estudio tiene una media de edad alrededor de los 20 años $(7,20)$.

Si bien es cierto que el patrón de consumo de tabaco se ha incrementado también es cierto que en lo que respecta al consumo de drogas el comportamiento es similar; la comparación de los datos obtenidos con estadísticas nacionales (2.5\%) solo hacen evidente este hecho, los porcentajes de consumidores activos de drogas son cada vez mayores, independientemente de la zona geográfica y del sexo (7). En cuanto al tipo de droga todos los consumidores activos y cerca de la totalidad de los exconsumidores refirieron el consumo de marihuana, situación esperada al ser la droga ilícita con mayor prevalencia de consumo a nivel mundial y nacional (4). Asimismo, la edad de inicio de 
consumo de droga en los estudiantes de ambos estados fue menor a la media nacional que es de 18.8 años $(7,13)$.

Otro dato relevante que vale la pena señalar tiene que ver con el estatus de consumidor y las personas del entorno de los jóvenes que consumen tabaco y/o drogas; en nuestro estudio, las personas de su red que consumen tabaco o que consumen drogas, en todos los casos fueron los amigos, en especial en los perfiles de fumador activo y exfumador; ello concuerda con el estudio realizado por Nebot y cols., (2002) en el cual se refiere que un factor determinante en el consumo es la norma social y la presión por parte de amigos, del mismo modo coincide con otro estudio hecho en México por Urquieta y cols., en el que refiere que la existencia de otros jóvenes en su red que fuman influye en la probabilidad de consumir tabaco (21-24).

Respecto al consumo de drogas ilícitas, la marihuana es la de mayor consumo, lo que también coincide con el reporte del SISVEA de 2013 en el cual la mayor frecuencia de consumo de marihuana se observó entre los 14 y 35 años con una prevalencia mucho menor de otro tipo de drogas ilícitas. Así mismo la mayor parte de los estudiantes casados y en unión libre se encontraron en el perfil de los que nunca han fumado lo cual corresponde a lo esperado al ser la marihuana como la principal droga de impacto en solteros. Nuevamente entre las personas de su red que consumen tabaco o que consumen drogas sobresalieron los amigos, en especial en los perfiles de consumidor activo y exconsumidor, similar a lo publicado por Medica-Mora y cols., que refiere una acelerada apertura social y cultural que ha afectado el ámbito individual y social en que interviene el abuso de sustancias $(4,18,24)$.

Es innegable que los cambios sociales que se han dado en las últimas décadas, originados de las transformaciones en la dinámica y composición de la estructura demográfica de la población, las crisis económicas y una acelerada apertura social y cultural frente al proceso de modernización y globalización, han afectado las vidas de las personas tanto en lo individual, como familiar y social. Los datos aquí analizados, en lo que al consumo de tabaco y drogas se refiere, si bien mostraron semejanzas entre estudiantes de las dos universidades, también es importante señalar que hay diferencias que habrá que tomar en cuenta al momento de las decisiones para elaborar e implementar programas de prevención sobre el abuso de sustancias. Los nuevos valores que se le transmite a este grupo de población, a través de los medios de comunicación, deben ser evaluados constantemente para evitar que se incorporen al mercado como bienes de consumo y sobre todo, adecuarlos a los contextos de los jóvenes. Por ello, por una parte es necesaria una política con relación al consumo de tabaco y drogas que busque disminuir los costos sociales que genera su uso y adoptar medidas urgentes de carácter público y social que encaminen la lucha contra estas adicciones de manera 
Sánchez-Hoil et al.

integral con promoción hacia la salud en la población juvenil que es altamente influenciada por los estilos de vida modernos y los medios de comunicación y por otra parte continuar con la generación de información que sirva de insumos para la elaboración de los programas de prevención.

\section{Agradecimientos}

Al proyecto Educación y Desarrollo Humano a partir de la Calidad de Vida y la Salud. Análisis de las campañas publicitarias (spots) e impacto de las mismas en estudiantes universitarios de los estados de Yucatán, Zacatecas y Coahuila. Etapa II financiado por el Programa del Mejoramiento al Profesorado (PROMEP) como parte de la vinculación interinstitucional de tres universidades: Yucatán, Zacatecas y Coahuila, en una Red Temática "Educación y Desarrollo Humano: Calidad de Vida y Salud"

A los Cuerpos Académicos "Salud, Ambiente y Desarrollo Humano" del Centro de Investigaciones Regionales "Dr Hideyo Noguchi” de la Universidad Autónoma de Yucatán y "Desarrollo Humano" de la Universidad Autónoma de Coahuila.

\section{Referencias}

1. Instituto Nacional de Salud Pública. Encuesta Nacional de Adicciones 2011: tabaco. México: INSP; 2013

2. Instituto Nacional de Salud Pública. Encuesta Nacional de Salud y Nutrición 2012. Disponible en:http//www.insp.mx/producción-editorial/novedades-editoriales/3057-ensanut2012-resultadosentidad-federativa.html.

3. Villatoro J, Medina-Mora ME, Fleiz C, Moreno M, Oliva N, Bustos M, et al. El consumo de drogas en México: Resultados de la Encuesta Nacional de Adicciones, 2011. Salud Mental 2012 Nov./Dic.; 35(6):447-457

4. Secretaría de Salud. Dirección General de Epidemiología. Sistema de Vigilancia Epidemiológica de las Adicciones (SISVEA): Informe 2011. México: Secretaría de Salud; 2013

5. World Health Organization. WHO Report on the Global Tobbaco Epidemic. Italy: WHO; 2011.

6. Unites Nations Office On Drugs And Crime. UNODC World Drug Report Vienna: United Nations publication; 2013.

7. De Micheli A \& Izaguirre R. Tabaco y tabaquismo en la historia de México y de Europa. Rev. invest. clin 2005 Ago; 57(4): 608-613.

8. Rodgman A, Perfetti TA. The Chemical components of tobbaco and tobbaco smoke. 2a ed. Estados Unidos: Taylor and Francis group; 2013.

9. Martínez MJ. El discurso social sobre drogas en la prensa de Murcia. $1^{\text {a }}$ ed. España: EDITUM; 2000

10. Maraj S, Figueredo VM, Lynn Morris D. Cocaine and the heart. Clin Cardiol. 2010 May; 33(5): 264269

11. Restrepo J. Corporalidades, Feminidades y Drogas Proscritas. Cult.drog. 2011 Oct; 16 (18): 225-235

12. Instituto Nacional de Psiquiatría Ramón de la Fuente Muñiz; Instituto Nacional de Salud Pública; Secretaría de Salud. Encuesta Nacional de Adicciones 2011: Reporte de drogas. México: INPRFM; 
2012. Disponible en: www.inprf.gob.mx,www.conadic.gob.mx,www.cenadic.salud.gob.mx, www.insp.mx

13. Comisión Nacional Contra las Adicciones. Análisis del consumo de sustancias en México: Generalidades de los opiáceos. México; CONACID. Disponible en: http://www.conacid.salud.gob.mx/pdfs/informe_opiaceos.pdf (citado el 14 de abril de 2015)

14. Rodríguez J. Redes en la era del conocimiento. Impacto social de la ciencia y la tecnología en el siglo XXI. Colección comunicaciones sociales, siglo XXI. Nicaragua. UAZ-Universidad de Nicaragua; 2012

15. Consejo Nacional contra las Adicciones, Instituto Nacional de Psiquiatría Ramón de la Fuente Muñiz, Instituto Nacional de Salud Pública, Secretaría de Salud, Fundación Gonzalo Río Arronte. Encuesta Nacional de adicciones 2008. Cuernavaca, México: INSP, 2009

16. Secretaría de Salud, Dirección General de Epidemiología. Encuesta Nacional de Adicciones 2002(ENA-2002). México; 2003.

17. Medina-Mora M, Natera G, Borges G, CraviotoP, Fleiz C, Tapia-Conyer R. Del siglo XX al tercer milenio. Las adicciones y la salud pública: drogas, alcohol y sociedad. Salud Mental 2001 Ago; 24(4):3-19.

18. Jha P, Ramasundarahettige C, Landsman V, Rostron B, Thun M, Anderson R, et al. $21^{\text {st }}$ Century Hazards of Smoking and Benefits of Cessation in the United States. New England Journal of Medicine 2013 Jan.; 368 (4): 341-350

19. Nazal-Beutelspacher A, Tapia-Conyer R, Villa-Romero A, León-Alvarez G, Medina-Mora M, Salvatierra-Izaba B. Factores asociados al consumo de Drogas en adolescentes de áreas urbanas de México. Salud Pública Mex. 1994 Nov./Dic.; 36(6):646-654

20. Organización Mundial de la Salud. Décima revisión de la Clasificación Internacional de Las Enfermedades. Trastornos Mentales y del Comportamiento: Descripciones Clínicas y pautas para el Diagnóstico CIE 10. Madrid; OMS, Meditor; 1992.

21. Urquieta JE, Hernández-Ávila M, Hernández B. El consumo de tabaco y alcohol en jóvenes de zonas marginadas de México. Un análisis de decisiones relacionadas. Salud Pública Mex. 2006 Abr.; 48 (suppl 1): s30-s40

22. Nebot M, Tomás Z, Ariza C, Valmayor S, Mudde A. Factores asociados con la intención de fumar y el inicio del hábito tabáquico en escolares: resultados del estudio ESFA en Barcelona. Gac Sanit 2002 Mar./Abr.;16(2):131-138

23. Nerín de la Puerta I, Marqueta A, Jiménez -Muro A, Gargallo P, Beamonte A, Consumo de drogas en estudiantes universitarios de primer curso. Adicciones [en línea] 2009 Enero [Fecha de acceso 24 de mayo 2016] Disponible en http://www.redalyc.org/artículo.oa?id=289122882004.

24. Cáceres D, Salazar I, Varela M, Tovar J. Consumo de drogas en jóvenes universitarios y su relación de riesgo y protección con los factores psicosociales. Univ.Psychol. 2006 oct./dic.; 5(3): 521- 534. 
Sánchez-Hoil et al.

Cuadro 1.- Contexto de estudiantes universitarios de YUCATÁN/COAHUILA

\begin{tabular}{|c|c|c|c|c|}
\hline \multicolumn{2}{|l|}{ Característica } & Yucatán & Coahuila & $\mathbf{P}$ \\
\hline \multicolumn{2}{|l|}{ Media de edad } & 20.64 & 19.55 & $<0.01$ \\
\hline \multirow[t]{2}{*}{ Sexo } & H & $257(55 \%)$ & $129(37.9 \%)$ & \multirow{2}{*}{$<0.01$} \\
\hline & M & $210(45 \%)$ & $211(62.1 \%)$ & \\
\hline \multirow[t]{2}{*}{ Estado civil } & Casado y unión libre & $10(2.1 \%)$ & $23(6.8 \%)$ & \multirow[t]{2}{*}{$<0.01$} \\
\hline & Soltero & 457 (97.9\%) & $317(93.2 \%)$ & \\
\hline \multicolumn{2}{|c|}{ Medias de personas de su red consumidoras de tabaco } & 2.25 & 3.34 & $<0.01$ \\
\hline \multicolumn{2}{|c|}{ Media de personas de su red consumidoras de droga } & 0.8 & 0.87 & 0.33 \\
\hline
\end{tabular}

\section{Cuadro 2.- Estatus del consumo de tabaco en universitarios de Yucatán y} Coahuila.

\begin{tabular}{|c|c|c|c|c|}
\hline Característica & & Yucatán & Coahuila & $\mathbf{P}$ \\
\hline \multirow{3}{*}{ Estatus de consumo de tabaco } & Fumador activo & $19.50 \%$ & $28.20 \%$ & \multirow[t]{3}{*}{$<0.01$} \\
\hline & Exfumador & $10.90 \%$ & $15.60 \%$ & \\
\hline & Nunca ha fumado & $69.60 \%$ & $56.20 \%$ & \\
\hline \multirow{3}{*}{$\begin{array}{l}\text { Estatus de consumo de tabaco en } \\
\text { hombres }\end{array}$} & Fumador activo & $19.80 \%$ & $34.90 \%$ & \multirow[t]{3}{*}{$<0.01$} \\
\hline & Exfumador & $11.30 \%$ & $17.80 \%$ & \\
\hline & Nunca ha fumado & $68.90 \%$ & $47.30 \%$ & \\
\hline \multirow{3}{*}{$\begin{array}{l}\text { Estatus de consumo de tabaco en } \\
\text { mujeres }\end{array}$} & Fumador activo & $19.00 \%$ & $24.20 \%$ & \multirow[t]{3}{*}{0.15} \\
\hline & Exfumador & $22.00 \%$ & $14.20 \%$ & \\
\hline & Nunca ha fumado & $70.00 \%$ & $61.60 \%$ & \\
\hline \multirow{3}{*}{$\begin{array}{c}\text { Cigarrillos por día en fumador } \\
\text { activo }\end{array}$} & Media general & 1.52 & 2.41 & $<0.01$ \\
\hline & Media en hombres & 1.47 & 2.91 & $<0.01$ \\
\hline & Media en mujeres & 1.58 & 1.98 & 0.24 \\
\hline \multirow{3}{*}{ Cigarrillos por día en exfumadores } & Media general & 1.26 & 3.26 & 0.01 \\
\hline & Media en hombres & 1.11 & 1.82 & 0.02 \\
\hline & Media en mujeres & 1.45 & 4.2 & 0.07 \\
\hline \multirow{3}{*}{ Razón para dejar el tabaco } & Salud & $66.60 \%$ & $71.60 \%$ & \multirow[t]{3}{*}{0.83} \\
\hline & Economía & $7.90 \%$ & $5.60 \%$ & \\
\hline & Otros & $25.50 \%$ & $22.80 \%$ & \\
\hline \multirow{3}{*}{$\begin{array}{l}\text { Edad de inicio en fumador activo y } \\
\text { exfumador }\end{array}$} & Media general & 17.18 & 16.15 & $<0.01$ \\
\hline & Media en hombres & 16.98 & 16.11 & $<0.01$ \\
\hline & Media en mujeres & 17.44 & 16.18 & $<0.01$ \\
\hline
\end{tabular}


Cuadro 3.- Perfil sociodemográfico de universitarios de Yucatán y Coahuila según su estatus de consumo de tabaco.

\begin{tabular}{|c|c|c|c|c|}
\hline \multicolumn{2}{|l|}{ Fumadores activos } & Yucatán & Coahuila & p \\
\hline \multicolumn{2}{|l|}{ Media de edad } & 20.44 & 19.62 & $<0.01$ \\
\hline \multirow[t]{2}{*}{ Sexo } & $\mathbf{H}$ & $51(56 \%)$ & $45(46.9 \%)$ & \multirow[t]{2}{*}{0.21} \\
\hline & M & $40(44 \%)$ & $51(53.1 \%)$ & \\
\hline \multirow[t]{2}{*}{ Estado civil } & Casado y unión libre & $3(3.3 \%)$ & $3(3.1 \%)$ & \multirow[t]{2}{*}{0.94} \\
\hline & Soltero & $88(96.7 \%)$ & $93(96.9 \%)$ & \\
\hline \multicolumn{2}{|c|}{ Media de personas de su red consumidoras de tabaco } & 3.02 & 3.79 & $<0.01$ \\
\hline \multicolumn{2}{|c|}{ Media de personas de su red consumidoras de droga } & 1.21 & 1.1 & 0.5 \\
\hline \multicolumn{5}{|l|}{ Exfumadores } \\
\hline \multicolumn{2}{|l|}{ Media de edad } & 20.78 & 21.03 & 0.4 \\
\hline \multirow[t]{2}{*}{ Sexo } & $\mathbf{H}$ & $29(56.9 \%)$ & $23(43.4 \%)$ & \multirow[t]{2}{*}{0.17} \\
\hline & M & $22(43.1 \%)$ & 30 (56.6\%) & \\
\hline \multirow[t]{2}{*}{ Estado civil } & Casado y unión libre & $3(5.9 \%)$ & $6(11.3 \%)$ & \multirow[t]{2}{*}{0.31} \\
\hline & Soltero & 48 (94.1\%) & 47 (88.7\%) & \\
\hline \multicolumn{2}{|c|}{ Media de personas de su red consumidoras de tabaco } & 2.63 & 3.58 & $<0.01$ \\
\hline \multicolumn{2}{|c|}{ Media de personas de su red consumidoras de droga } & 1.06 & 1 & 0.72 \\
\hline \multicolumn{5}{|l|}{ Nunca han fumado } \\
\hline \multicolumn{2}{|l|}{ Media de edad } & 20.67 & 19.1 & $<0.01$ \\
\hline \multirow[t]{2}{*}{ Sexo } & $\mathbf{H}$ & $177(54.5 \%)$ & $61(31.9 \%)$ & \multirow[t]{2}{*}{$<0.01$} \\
\hline & M & $148(45.5 \%)$ & $130(68.1 \%)$ & \\
\hline \multirow[t]{2}{*}{ Estado civil } & Casado y unión libre & $4(1.2 \%)$ & $14(7.3 \%)$ & \multirow[t]{2}{*}{$<0.01$} \\
\hline & Soltero & $321(98.8 \%)$ & $177(92.7 \%)$ & \\
\hline \multicolumn{2}{|c|}{ Media de personas de su red consumidoras de tabaco } & 1.98 & 3.04 & $<0.01$ \\
\hline \multicolumn{2}{|c|}{ Media de personas de su red consumidoras de droga } & 0.64 & 0.72 & 0.35 \\
\hline \multirow{3}{*}{$\begin{array}{l}\text { Frecuencia de consumo de tabaco en } \\
\text { personas de su red }\end{array}$} & Padre & $43(47.3 \%)$ & $43(44.8 \%)$ & \\
\hline & Primos & $37(40.7 \%)$ & $58(60.4 \%)$ & \\
\hline & Amigos & $75(82.4 \%)$ & $73(76 \%)$ & \\
\hline
\end{tabular}


Sánchez-Hoil et al.

Cuadro 4.- Estatus del consumo de droga en universitarios de Yucatán y Coahuila.

\begin{tabular}{|c|c|c|c|c|}
\hline Característica & & Yucatán & Coahuila & $\mathbf{P}$ \\
\hline \multirow{3}{*}{ Estatus de consumo de droga } & Consumidor activo & $5.8 \%$ & $9.7 \%$ & \multirow[t]{3}{*}{0.06} \\
\hline & Exconsumidor & $6.6 \%$ & $8.2 \%$ & \\
\hline & Nunca ha consumido & $87.6 \%$ & $81.5 \%$ & \\
\hline \multirow{3}{*}{ Estatus de consumo de droga en hombres } & Consumidor activo & $7 \%$ & $12.4 \%$ & \multirow[t]{3}{*}{0.04} \\
\hline & Exconsumidor & $9.3 \%$ & $14.7 \%$ & \\
\hline & Nunca ha consumido & $83.7 \%$ & $71.9 \%$ & \\
\hline \multirow{3}{*}{ Estatus de consumo de droga en mujeres } & Consumidor activo & $4.3 \%$ & $8.1 \%$ & \multirow[t]{3}{*}{0.05} \\
\hline & Exconsumidor & $3.3 \%$ & $4.3 \%$ & \\
\hline & Nunca ha consumido & $92.4 \%$ & $86.7 \%$ & \\
\hline \multirow{2}{*}{$\begin{array}{c}\text { Tipo de droga en activo } \\
\text { Tipo de droga en exconsumidor }\end{array}$} & Marihuana & $100 \%$ & $100 \%$ & \\
\hline & Marihuana & $96.8 \%$ & $92.8 \%$ & \\
\hline \multirow{3}{*}{ Razón para dejar el consumo de droga } & Salud & $83.8 \%$ & $46.4 \%$ & \multirow[t]{3}{*}{$<0.01$} \\
\hline & Economía & $3.2 \%$ & $10.7 \%$ & \\
\hline & Otros & $13 \%$ & $42.9 \%$ & \\
\hline \multirow{3}{*}{$\begin{array}{l}\text { Edad de inicio en consumidor activo y } \\
\text { exconsumidor }\end{array}$} & Media general & 17.9 & 16.67 & $<0.01$ \\
\hline & Media en hombres & 17.81 & 16.57 & $<0.01$ \\
\hline & Media en mujeres & 18.13 & 16.8 & 0.04 \\
\hline
\end{tabular}


Cuadro 5.- Perfil sociodemográfico de universitarios de Yucatán y de Coahuila según su estatus de consumo de droga.

\begin{tabular}{|c|c|c|c|c|}
\hline \multicolumn{2}{|l|}{ Consumidores activos } & Yucatán & Coahuila & $\mathbf{P}$ \\
\hline \multicolumn{2}{|l|}{ Media de edad } & 20.22 & 19.51 & 0.02 \\
\hline \multirow[t]{2}{*}{ Sexo } & $\mathbf{H}$ & $18(66.7 \%)$ & $16(48.5 \%)$ & \multirow[t]{2}{*}{0.16} \\
\hline & M & $9(33.3 \%)$ & $17(51.5 \%)$ & \\
\hline \multirow[t]{2}{*}{ Estado civil } & Casado y unión libre & $1(3.7 \%)$ & $3(9.1 \%)$ & \multirow{2}{*}{0.4} \\
\hline & Soltero & $26(96.3 \%)$ & 30 (90.9\%) & \\
\hline \multirow[t]{2}{*}{ Campus } & Ciencias sociales & $10(62.5 \%)$ & $21(63.6 \%)$ & \multirow[t]{2}{*}{0.93} \\
\hline & Ciencias exactas & $6(37.5 \%)$ & $12(36.4 \%)$ & \\
\hline \multicolumn{2}{|c|}{ Media de personas de su red consumidoras de tabaco } & 3.56 & 4.03 & 0.31 \\
\hline \multicolumn{2}{|c|}{ Media de personas de su red consumidoras de droga } & 1.81 & 1.75 & 0.86 \\
\hline \multicolumn{5}{|l|}{ Exconsumidores } \\
\hline \multicolumn{2}{|l|}{ Media de edad } & 20.61 & 19.5 & 0.09 \\
\hline \multirow[t]{2}{*}{ Sexo } & $\mathbf{H}$ & $24(77.4 \%)$ & $19(67.9 \%)$ & \multirow[t]{2}{*}{0.41} \\
\hline & M & $7(22.6 \%)$ & $9(32.1 \%)$ & \\
\hline \multirow[t]{2}{*}{ Estado civil } & Casado y unión libre & $1(3.2 \%)$ & $2(7.1 \%)$ & \multirow[t]{2}{*}{0.49} \\
\hline & Soltero & $30(96.8 \%)$ & 26 (92.9\%) & \\
\hline \multirow[t]{2}{*}{ Campus } & Ciencias sociales & $9(39.1 \%)$ & $17(60.7 \%)$ & \multirow[t]{2}{*}{0.12} \\
\hline & Ciencias exactas & $14(60.9 \%)$ & $11(39.3 \%)$ & \\
\hline \multicolumn{2}{|c|}{ Media de personas de su red consumidoras de tabaco } & 2.61 & 4.03 & $<0.01$ \\
\hline \multicolumn{2}{|c|}{ Media de personas de su red consumidoras de droga } & 1.55 & 1.64 & 0.73 \\
\hline \multicolumn{5}{|l|}{ Nunca ha consumido } \\
\hline \multicolumn{2}{|l|}{ Media de edad } & 20.67 & 19.54 & $<0.01$ \\
\hline \multirow[t]{2}{*}{ Sexo } & $\mathbf{H}$ & $215(52.6 \%)$ & $94(33.9 \%)$ & \multirow[t]{2}{*}{$<0.01$} \\
\hline & M & $194(47.4 \%)$ & $183(66.1 \%)$ & \\
\hline \multirow[t]{2}{*}{ Estado civil } & Casado y unión libre & $8(2 \%)$ & $18(6.5 \%)$ & \multirow[t]{2}{*}{$<0.01$} \\
\hline & Soltero & $401(98 \%)$ & $259(93.5 \%)$ & \\
\hline \multicolumn{2}{|c|}{ Media de personas de su red consumidoras de tabaco } & 2.14 & 3.19 & $<0.01$ \\
\hline \multicolumn{2}{|c|}{ Media de personas de su red consumidoras de droga } & 0.67 & 0.69 & 0.76 \\
\hline \multirow[t]{2}{*}{$\begin{array}{l}\text { Frecuencia de consumo de drogas en } \\
\text { personas de su red }\end{array}$} & Primos & $11(40.7 \%)$ & $15(45.5 \%)$ & \\
\hline & Amigos & $21(77.8 \%)$ & 23 (69.7\%\%) & \\
\hline
\end{tabular}

